\title{
Self-consistent model for ambipolar tunneling in quantum-well systems
}

\author{
C. Presilla, V. Emiliani and A. Frova \\ Dipartimento di Fisica, Università di Roma "La Sapienza", \\ Piazzale A. Moro 2, Roma, Italy 00185 \\ (Semiconductors Science \& Technology 10 (1995) 577-585)
}

\begin{abstract}
We present a self-consistent approach to describe ambipolar tunneling in asymmetrical double quantum wells under steady-state excitation and extend the results to the case of tunneling from a near-surface quantum well to surface states. The results of the model compare very well with the behavior observed in photoluminescence experiments in In GaAs/InP asymmetric double quantum wells and in near-surface $A l G a A s / G a A s$ single quantum wells.
\end{abstract}

73.20.Dx, 73.40.Gk, 78.65.Fa

\section{INTRODUCTION}

Tunneling of electrons and holes in quasi-two-dimensional semiconductor heterostructures is the object of large interest both for its physical interest - it is one of the most important quantum-mechanical effect observed in lowdimensional structures - and for its role in several nanometric devices and applications. Much work has been done concerning tunneling in symmetrical and asymmetrical double quantum wells (ADQW).

Tunneling from a quantum well $(\mathrm{QW})$ to surface states has received instead little attention, despite the great importance of gaining control over this mechanism. For a quantum well built in the neighborhood of an unpassivated surface, an extra non-radiative recombination channel becomes available to electrons and holes if they can tunnel to surface states, with consequent loss in emission efficiency from the quantum well. The importance of this effect has been demonstrated experimentally in recent papers and its dependence on surface-barrier thickness investigated [1 4 .

In the interpretation of the experimental results, both tunneling to surface states and tunneling between ADQW, a problem arises. Although in some cases hole-tunneling rates can be comparable to the electronic ones, e.g. when heavy holes in one well move to a light hole state in the other well [5], direct-gap III-V semiconductors have in general rather different tunneling probabilities for electrons and holes due to the different effective masses [6.,7]. The different tunneling probability for the two carriers causes a dipole electric field across the tunneling barrier to develop [8 11], so as to induce - via the quantum confined Stark effect [12] - a peak shift of the excitonic recombination, and to affect the tunneling probabilities towards an ambipolar regime, with equal tunneling currents for electrons and holes.

The appearance of an electric field due to the spatial separation of electrons and holes in ADQW systems was already discussed theoretically [13] in the framework of exciton tunneling under impulsive excitation. In this paper we study the tunneling of unpaired electrons and holes both in ADQW and in near-surface QW systems under steadystate excitation. Unlike the case of impulsive excitation, not discussed in this paper, the ambipolar regime is shown to be reached in this case for any excitation intensity. The consequently modified tunneling properties are successfully compared with available experiments, i.e. dependence of the emission efficiency ratio in ADQW systems on the excitation power and the tunneling-barrier width [9], dependence of the tunneling current on the barrier thickness [1], and dependence of the Stark shift on the excitation level in a near-surface QW [8].

\section{TUNNELING BETWEEN TWO ASYMMETRIC QUANTUM WELLS}

Here we describe the steady-state photoluminescence from two coupled asymmetric quantum wells under constant irradiation. The charges photogenerated inside the wells relax almost instantaneously with respect to the other relevant time scales to the lowest band of the respective well and fill it according to the Pauli principle. Electron-hole interaction leads to exciton formation. Tunneling between the two wells is essentially restricted to unpaired electrons and holes when the tunneling process is a small perturbation for the nearly uncoupled wells: as a matter of fact, the tunneling current densities correspond to a first-order process for unpaired electrons and holes and to a second-order process for excitons. On the other hand photoluminescence is restricted only to excitonic recombination.

Let $a_{1}$ and $a_{2}$ be the widths of the two quantum wells and $b$ the width of the barrier between them. We suppose $a_{1}>a_{2}$ so that the bottom of the $e 1$ and $h h 1$ bands of well $2, E_{e 1}^{(2)}$ and $E_{h h 1}^{(2)}$, are higher in energy than those of well 
$1, E_{e 1}^{(1)}$ and $E_{h h 1}^{(1)}$. Let $G_{1}$ and $G_{2}$ be the generation current densities of electron-hole pairs in the two wells. If $n_{i}$ and $p_{i}$ are the steady-state concentrations (number of particles per unit area) of electrons and holes in the well $i=1,2$, the following rate equations hold:

$$
\begin{gathered}
0=G_{1}+J_{e}-\lambda_{1} n_{1} p_{1} \\
0=G_{1}+J_{h}-\lambda_{1} n_{1} p_{1} \\
0=G_{2}-J_{e}-\lambda_{2} n_{2} p_{2} \\
0=G_{2}-J_{h}-\lambda_{2} n_{2} p_{2} \\
0=\lambda_{1} n_{1} p_{1}-I_{1} \\
0=\lambda_{2} n_{2} p_{2}-I_{2} .
\end{gathered}
$$

The first two couples of equations are the rate equations for the concentrations of unpaired electrons and holes in well 1 and 2, respectively, and contain, in the order, the photogeneration and tunneling current densities of the unpaired charges and the generation current density of excitons. The last two equations are the rate equations for the exciton concentrations in well 1 and 2 and contain the photoluminescence current densities $I_{1}$ and $I_{2}$ which are the quantities we want to evaluate and compare with experimental results.

The generation current density of excitons in well $i$ is assumed proportional to the concentrations of the unpaired charges in the same well, $\lambda_{i} n_{i} p_{i}$, where $\lambda_{i}$ is the bimolecular exciton formation coefficient possibly dependent on the well size [14].

A little more complicated analysis is needed for the tunneling current densities $J_{e}$ and $J_{h}$. Transfer of electrons (holes) from the narrower well to the larger one is realized in a non-coherent two-step process. Quantum coherent tunneling of electrons (holes) from an occupied state of the $e 1$ ( $h h 1$ ) band of well 2 to an equal-energy empty state of the $e 1$ ( $h h 1)$ band of well 1 is followed by thermalization toward the lower energy states of well 1 . When the barrier width $b$ is not too small, which is also the range of validity of the above rate equations, the quantum coherent tunneling process characterized by a time growing exponentially with $b$ gets much slower than the thermalization process in well $1(\lesssim 1$ ps $[15])$ and this last can be neglected.

The current densities for quantum coherent tunneling are approximately proportional to the charge concentrations in well 2 and the proportionality factor, namely the tunneling rate, is quite different for electrons and holes due to their different effective masses. Therefore, in a steady-state situation when $J_{e}=J_{h}$, the concentrations of electrons and holes in each single well must be different and an electric field gets established in the barrier between the two wells. Moreover the electric field modifies the electron and hole tunneling rates. The direction of the field is simply understood from the values of the tunneling rates at zero field. As electron tunneling rate is expected in this case to be much greater than the hole tunneling rate, electrons accumulate at well 1 and in a steady-state situation $n_{1}>p_{1}$. The electric field is directed from well 2 to well 1 and its value is given by

$$
F=\frac{e n}{\varepsilon_{0} \varepsilon_{r}}
$$

where $n=n_{1}-p_{1}=p_{2}-n_{2}$ and $\varepsilon_{r}$ is the permittivity of the barrier material.

The tunneling current densities depend on the electric field $F$ through the dependence of the charge concentrations on $F$ as well as through the dependence of the tunneling rate on the energy shift between the bands of the two wells induced by $F$. Assuming that the electric field is completely shielded inside the wells, this energy shift amounts to $e F b$. For a given value of $F$, the tunneling current densities can be evaluated within perturbation theory in the case of a barrier not too thin. At the first order the transition rate induced by a constant perturbation $V$ between initial and final states continuously distributed in energy with densities $d N_{i} / d \epsilon$ and $d N_{f} / d \epsilon$, respectively, is [16]

$$
\iint \frac{2 \pi}{\hbar}\left|\left\langle i, \epsilon|V| f, \epsilon^{\prime}\right\rangle\right|^{2} \delta\left(\epsilon-\epsilon^{\prime}\right) d N_{f}\left(\epsilon^{\prime}\right) d N_{i}(\epsilon)=\int \frac{2 \pi}{\hbar}|\langle i, \epsilon|V| f, \epsilon\rangle|^{2} \frac{d N_{f}}{d \epsilon}(\epsilon) \frac{d N_{i}}{d \epsilon}(\epsilon) d \epsilon
$$

This formula, when applied to the electron and hole states of the two uncoupled wells and divided by the transverse area $A$ of the heterostructure, gives the following expressions for the tunneling current densities 


$$
\begin{gathered}
J_{e}=\frac{1}{A} \int_{0}^{\infty} \frac{2 \pi}{\hbar}\left|\left\langle\Phi_{\epsilon}^{(1)}\left|V_{e}\right| \Phi_{\epsilon}^{(2)}\right\rangle\right|^{2} A \nu_{e}^{(1)}\left[1-f\left(\frac{\epsilon-\epsilon_{F}^{(1)}}{k_{B} T}\right)\right] A \nu_{e}^{(2)} f\left(\frac{\epsilon-\epsilon_{F}^{(2)}}{k_{B} T}\right) d \epsilon \\
J_{h}=\frac{1}{A} \int_{0}^{\infty} \frac{2 \pi}{\hbar}\left|\left\langle\Phi_{\epsilon}^{(1)}\left|V_{h}\right| \Phi_{\epsilon}^{(2)}\right\rangle\right|^{2} A \nu_{h}^{(1)}\left[1-f\left(\frac{\epsilon-\epsilon_{F}^{(1)}}{k_{B} T}\right)\right] A \nu_{h}^{(2)} f\left(\frac{\epsilon-\epsilon_{F}^{(2)}}{k_{B} T}\right) d \epsilon .
\end{gathered}
$$

The integration takes into account the transitions from all the occupied states in the $e 1(h h 1)$ band of well 2 (initial states) to the empty states in the $e 1$ ( $h h 1)$ band of well 1 (final states) at the same energy $\epsilon$ measured from the bottom of $e 1(h h 1)$ band of well 2. The densities of the available initial (final) states are obtained by multiplying the number of states per unit energy $A \nu^{(2)}\left(A \nu^{(1)}\right)$ with the appropriate occupation probability. Here $\nu_{e}^{(i)}=m_{e}^{(i)} / \pi \hbar^{2}$ $\left(\nu_{h}^{(i)}=m_{h}^{(i)} / \pi \hbar^{2}\right), \mathrm{i}=1,2$, are the densities per unit area and unit energy of a two dimensional ideal gas of fermions with mass $m_{e}^{(i)}\left(m_{h}^{(i)}\right)$ and $f(x)=1 /[\exp (x)+1]$ is the Fermi function. The Fermi energies for electrons (holes) are related to the concentrations in the corresponding well: $\epsilon_{F}^{(1)}=\pi \hbar^{2} n_{1} / m_{e}^{(1)}+E_{e 1}^{(1)}+e F b-E_{e 1}^{(2)}$ and $\epsilon_{F}^{(2)}=\pi \hbar^{2} n_{2} / m_{e}^{(2)}$ $\left(\epsilon_{F}^{(1)}=\pi \hbar^{2} p_{1} / m_{h}^{(1)}+E_{h h 1}^{(1)}-e F b-E_{h h 1}^{(2)}\right.$ and $\left.\epsilon_{F}^{(2)}=\pi \hbar^{2} p_{2} / m_{h}^{(2)}\right)$. Finally, the perturbation potential $V_{e}\left(V_{h}\right)$ is the potential of the heterostructure ( $b$ finite) coupling the electron (hole) states evaluated by considering the wells uncoupled ( $b$ infinite). Due to the exponentially vanishing tails of the electron (hole) wavefunctions, the relevant contribution to the matrix elements comes only from the barrier region between the two wells [16]. In this region the potential $V_{e}\left(V_{h}\right)$ has magnitude of the order of the conduction (valence) band offset $\Delta E_{c}\left(\Delta \overline{E_{v}}\right)$. More accurate expressions for $V_{e}\left(V_{h}\right)$, e.g. taking into account the distortion due to the electric field, have little influence on the final result and will be neglected. Explicit expressions of the electron and hole states $\Phi$ and analytical evaluation of the matrix elements are given in Appendix A.

The formulas for the tunneling current densities can be simplified by noting that, due to the exponentially large difference between electron and hole tunneling rates, we expect $n_{2} \ll n_{1}$. As a consequence, the electron Fermi energy $\epsilon_{F}^{(2)}$, i.e. the effective region of integration in Eq. (4), is very small. By approximating all factors to be integrated in (任) but the Fermi function in well 2 with their value at $\epsilon=0$ (the bottom of the $e 1$ band of well 2) we get $J_{e}=n_{2} / \tau_{e}$, where $n_{2}=\int \nu_{e}^{(2)} f\left(\left(\epsilon-\epsilon_{F}^{(2)}\right) / k_{B} T\right) d \epsilon$ and

$$
\frac{1}{\tau_{e}}=\frac{2 \pi}{\hbar}\left|\left\langle\Phi_{0}^{(1)}\left|V_{e}\right| \Phi_{0}^{(2)}\right\rangle\right|^{2} A \nu_{e}^{(1)} f\left(\frac{\epsilon_{F}^{(1)}}{k_{B} T}\right) .
$$

In the case of holes we expect $p_{2} \gg p_{1}$ so that $\epsilon_{F}^{(2)}$ is not small. However, in this case $\epsilon_{F}^{(1)}<0$ (now the zero of energy is the bottom of the $h h 1$ band of well 2) and $f\left(\left(\epsilon-\epsilon_{F}^{(1)}\right) / k_{B} T\right) \simeq 0$. Neglecting the smooth dependence of the matrix element on $\epsilon$ in (5) we get $J_{h}=p_{2} / \tau_{h}$ where $p_{2}=\int \nu_{h}^{(2)} f\left(\left(\epsilon-\epsilon_{F}^{(2)}\right) / k_{B} T\right) d \epsilon$ and

$$
\frac{1}{\tau_{h}}=\frac{2 \pi}{\hbar}\left|\left\langle\Phi_{0}^{(1)}\left|V_{h}\right| \Phi_{0}^{(2)}\right\rangle\right|^{2} A \nu_{h}^{(1)} .
$$

Note that both the tunneling rates $1 / \tau_{e}$ and $1 / \tau_{h}$ depend on the electric field through the matrix elements (see Appendix A). In addition the electron tunneling rate depends on $F$ through the Fermi energy of the well 1.

If we think that the electric field, i.e. $n$, and the tunneling rates are known, we get the following solution for the initial set of rate equations

$$
\begin{gathered}
n_{2}=\sqrt{\left(\frac{n}{2}+\frac{1}{2 \lambda_{2} \tau_{e}}\right)^{2}+\frac{G_{2}}{\lambda_{2}}}-\left(\frac{n}{2}+\frac{1}{2 \lambda_{2} \tau_{e}}\right) \\
n_{1}=\sqrt{\left(\frac{n}{2}\right)^{2}+\frac{G_{1}}{\lambda_{1}}+\frac{n_{2}}{\lambda_{1} \tau_{e}}}+\frac{n}{2} \\
p_{1}=n_{1}-n \\
p_{2}=n_{2}+n .
\end{gathered}
$$


This result allows us to find the steady-state values of the electric field, of the charge concentrations and of the tunneling rates by a recursive method. We proceed in the following manner. First of all we fix an electric field value corresponding to some negative electric charge concentration $n$ in the well 1 . Then we evaluate the hole tunneling rate and give a starting value to the electron tunneling rate (for instance that obtained for $n_{1}=n$ ) so we can deduce some charge concentrations by Eq. (8). From these concentrations we evaluate a new electron tunneling rate by Eq. (6) and from that new charge concentrations. After few iterations, the electron tunneling rate and the charge concentrations converge to the solution corresponding to the value of the electric field fixed at the beginning. At this point we compare the values of the tunneling current densities. If $J_{e}>J_{h}$ the electric field is increased, if $J_{e}<J_{h}$ the electric field is decreased. The procedure is repeated until the steady-state condition $J_{e}=J_{h}$ is reached. Note that the existence of the ambipolar regime does not rely upon the value of the excitation intensity, i.e. $G_{1}$ and $G_{2}$. However, the value of the electric field established in the steady state strongly depends on the excitation intensity.

We illustrate the behavior of the model by considering a practical example close to the experimental situation investigated by Sauer, Thonke and Tsang [9]. We consider two $I n_{0.53} G a_{0.47} A s$ quantum wells imbedded between $\operatorname{InP}$ barriers with $a_{1}=100 \AA$ and $a_{2}=60 \AA$. We use the following material parameters at $T=4.2 \mathrm{~K}[17]$ : $\Delta E_{c}=0.195$ $\mathrm{eV}, \Delta E_{v}=0.293 \mathrm{eV}, m_{e}^{(1)}=m_{e}^{(2)}=0.044 m, m_{h}^{(1)}=m_{h}^{(2)}=0.38 m, m$ being the free electron mass, and $\varepsilon_{r}=13.9$. Moreover we put $\lambda_{1}=\lambda_{2}=6 \mathrm{~cm}^{2} \mathrm{~s}^{-1}$ [1] . Since $\alpha a_{1}, \alpha a_{2} \ll 1$, where $\alpha$ is the optical absorption coefficient for the pump light, we take $G_{1} / G_{2}=a_{1} / a_{2}$ and $G_{2}=P / h \nu$ where $P$ is the absorbed power density and $h \nu=2.41 \mathrm{eV}$ is the photon energy corresponding to a 514-nm laser light. Note that the transverse area $A$ shown in Eq. (6) and Eq. (7) cancels out with the $A^{-1}$ from the squared matrix elements and is an irrelevant parameter.

In Fig. 1 we show the calculated photoluminescence intensity ratio $I_{1} / I_{2}$ from the two wells as a function of the absorbed power density $P$ for different values of the barrier width $b$. The self-consistent electric field $F$ generated between the two wells in the same cases is shown in Fig. 2. When $P$ is lower than a critical value, which depends on the barrier width, the photoluminescence intensity ratio $I_{1} / I_{2}$ approximately decreases as $P^{-1}$ and the electric field increases as $P$. When $P$ reaches and exceeds the critical value an electric field of the order of $3 \div 4 \times 10^{4} \mathrm{~V} \mathrm{~cm}{ }^{-1}$ gets established which slows down the tunneling of electrons from well 2 to well 1 and enhances the photoluminescence from well 2. At very high absorbed power density the ratio $I_{1} / I_{2}$ tends to the value $G_{1} / G_{2}$ for two uncoupled wells. These results compare quite well with the experimental findings [9] if one assumes a $0.1 \%$ excitation efficiency.

A deeper understanding of the behavior shown in Fig.s 1 and 2 can be reached by an approximate solution of the self-consistent procedure described above. Let us first concentrate on the dependence of the electric field, i.e. $n$, on the absorbed power density $P$. In the steady state we have $n_{2} / \tau_{e}=p_{2} / \tau_{h}$ which combined with Eq. (\$a) and Eq. (8)d) gives

$$
n=\frac{1}{2 \lambda_{2}}\left(\frac{1}{\tau_{e}}-\frac{1}{\tau_{h}}\right)\left(\sqrt{1+\frac{4 \lambda_{2} \tau_{e} \tau_{h}}{h \nu} P}-1\right) .
$$

When $P$ is not too high the second term in the squared root is small and we get the simple self-consistent equation

$$
n+\frac{\tau_{e}(n)}{h \nu} P=\frac{\tau_{h}}{h \nu} P .
$$

In a first approximation the smooth dependence of the tunneling rates on the electric field through the corresponding matrix elements can be neglected so that $\tau_{h}$ is constant while $\tau_{e}$ still depends on $n$ through the Fermi function. The argument of the Fermi function in Eq. (6) is negative until the alignment of the electron bands is achieved due to the electric field, i.e. in the region $n \leq n_{\text {crit }}$ where $e^{2} b n_{\text {crit }} / \epsilon_{0} \epsilon_{r}=E_{e 1}^{(2)}-E_{e 1}^{(1)}$. In this range $\tau_{e}$ is approximately constant and much smaller than $\tau_{h}$. On the other hand for $n>n_{\text {crit }}$, the electron tunneling time increases exponentially with the adimensional parameter $e F b / k_{B} T$, i.e. with $n$. The behavior of $\tau_{e}(n)$ imposes different solutions to Eq. (10) at different values of the absorbed power density. At low $P$ we have $\tau_{e}(n) P / h \nu \ll n$ and therefore $n \simeq \tau_{h} P / h \nu$. At high $P$ we have $\tau_{e}(n) P / h \nu \gg n$ and therefore $n \simeq n_{\text {crit }}$ is constant. The critical value of the electric field is related to the critical value of the absorbed power density by $P_{\text {crit }} \simeq h \nu n_{\text {crit }} / \tau_{h}$. Since $\tau_{h}$ increases exponentially with the barrier width $b, P_{\text {crit }}$ decreases exponentially with increasing $b$ in agreement with Fig. 2.

The behavior of $n(P)$ allows us to understand also the features of Fig. 1. By approximating Eq. (8a) with $n_{2} \simeq G_{2} \tau_{e} /\left(1+n \lambda_{2} \tau_{e}\right)$ we get

$$
I_{1} / I_{2}=\frac{\lambda_{1} n_{1}\left(n_{1}-n\right)}{\lambda_{2} n_{2}\left(n_{2}+n\right)} \simeq \frac{1}{n \lambda_{2} \tau_{e}}\left[1+\frac{G_{1}}{G_{2}}\left(1+n \lambda_{2} \tau_{e}\right)\right] .
$$

When $P \ll P_{\text {crit }}$ the electron tunneling time is almost constant, $n \lambda_{2} \tau_{e} \ll 1$ and the ratio $I_{1} / I_{2}$ decreases as $n^{-1}$, i.e. $P^{-1}$. The behavior of $I_{1} / I_{2}$ changes drastically at the critical power $P_{\text {crit }}$ due to the exponential change of $\tau_{e}$ and, finally, for $n \lambda_{2} \tau_{e} \gg 1$ the asymptotic value $I_{1} / I_{2}=G_{1} / G_{2}$ is obtained. 
For purpouse of comparison with the following Section, in Fig. 3 we show the behavior of the calculated normalized photoluminescence intensities $I_{i} / I_{i \infty}, \mathrm{i}=1,2$, in the same ADQW of Fig.s 1 and 2 as a function of the barrier width $b$ and for different absorbed powers. The normalization $I_{i \infty}$ is the photoluminescence current density for $b \rightarrow \infty$. The corresponding self-consistent electric field is shown in Fig. 4. The normalized photoluminescence intensity of well 2 Fig. 3 - vanishes at smaller $b$ when the tunneling current becomes higher. In this limit $J_{e} \rightarrow G_{2}$. At the same time the normalized photoluminescence intensity of well 1 tends to $\left(G_{1}+J_{e}\right) / G_{1}=1+G_{2} / G_{1}$. The self-consistent electric field - Fig. 4 - gradually increases for decreasing $b$, until, in the tunneling dominated limit $I_{2} / I_{2 \infty} \rightarrow 0$, it vanishes exponentially.

\section{TUNNELING FROM A QUANTUM WELL TO SURFACE STATES}

The considerations developed in the previous sections can be extended to describe the case where a single quantum well is close to a surface. This is another situation where tunneling followed by recombination takes place [1 4 , 8 . Electron and hole bands of defect states localized at the surface play the role of the $e 1$ and $h h 1$ bands of the missing well. In a steady-state situation the system can be still described by the rate equations (1) where the index 1 is associated with the surface and the index 2 with the well. Let $n_{1}$ and $p_{1}$ be the charge concentrations in the donorlike and acceptor-like surface bands. We can neglect the photogeneration of pairs at the surface so that $G_{1}=0$. Moreover $\lambda_{1} n_{1} p_{1}=I_{1}$ represents the nonradiative recombination current density at the surface. Since electrons and holes recombine (through a multiphonon process) very fast with respect to the other relevant time scales, one is allowed to take $\lambda_{1} \rightarrow \infty$.

The tunneling current densities from the occupied states of well 2 to the empty surface states 1 are still given by the general expressions (4) and (5). Now, however, the surface densities $\nu_{e}^{(1)}$ and $\nu_{h}^{(1)}$ are not constant in general. This implies the following definition for the corresponding Fermi energies:

$$
\int_{E_{e 1}^{(1)}+e F b-E_{e 1}^{(2)}}^{\epsilon_{F}^{(1)}} \nu_{e}^{(1)}(\epsilon) d \epsilon=n_{1}
$$

for the donor-like band, and

$$
\int_{E_{h h 1}^{(1)}-e F b-E_{h h 1}^{(2)}}^{\epsilon_{F}^{(1)}} \nu_{h}^{(1)}(\epsilon) d \epsilon=p_{1}
$$

for the acceptor-like band. As in the case of the two wells, we measure the energies from the bottom of the $e 1$ or $h h 1$ band of well 2. More importantly, $\nu_{e}^{(1)}$ and $\nu_{h}^{(1)}$ depend on the material characteristics and can be orders of magnitude different. If $\nu_{e}^{(1)} \ll \nu_{h}^{(1)}$, the effective mass difference between electrons and holes can be overcompensated by the difference in the surface densities and holes can tunnel more effectively than electrons do. This is the case, for instance, at the GaAs-oxide interface [8,18]. In this case holes accumulate at the surface and in a steady-state situation $p_{1}>n_{1}$. The electric field still given by Eq. (2) turns out to be negative.

As in the case of the two wells, the tunneling current densities can be approximated by $J_{e}=n_{2} / \tau_{e}$ and $J_{h}=p_{2} / \tau_{h}$. Now, however, we distinguish two cases. If $n_{1}>p_{1}$, the tunneling rates are given by Eq. (河) and Eq. (7) with $\nu_{e}^{(1)}=\nu_{e}^{(1)}(0)$ and $\nu_{h}^{(1)}=\nu_{h}^{(1)}(0)$. If $p_{1}>n_{1}$, we have

$$
\begin{gathered}
\frac{1}{\tau_{e}}=\frac{2 \pi}{\hbar}\left|\left\langle\Phi_{0}^{(1)}\left|V_{e}\right| \Phi_{0}^{(2)}\right\rangle\right|^{2} A \nu_{e}^{(1)}(0) \\
\frac{1}{\tau_{h}}=\frac{2 \pi}{\hbar}\left|\left\langle\Phi_{0}^{(1)}\left|V_{h}\right| \Phi_{0}^{(2)}\right\rangle\right|^{2} A \nu_{h}^{(1)}(0) f\left(\frac{\epsilon_{F}^{(1)}}{k_{B} T}\right) .
\end{gathered}
$$

When the electric field and the tunneling rates are known, the solution of the initial rate equations is still given by Eq. (8). With the conditions $G_{1}=0$ and $\lambda_{1} \rightarrow \infty$ we get the simpler formulas

$$
n_{2}=\sqrt{\left(\frac{n}{2}+\frac{1}{2 \lambda_{2} \tau_{e}}\right)^{2}+\frac{G_{2}}{\lambda_{2}}}-\left(\frac{n}{2}+\frac{1}{2 \lambda_{2} \tau_{e}}\right)
$$




$$
\begin{gathered}
n_{1}=n \\
p_{1}=0 \\
p_{2}=n_{2}+n .
\end{gathered}
$$

The same recursive method explained in section II, allows us to find the steady-state values of the electric field, of the charge concentrations and of the tunneling rates.

The evaluation of the luminescence intensity $I_{2}$ implies the knowledge of the nature of the surface states. We can try to get information on the surface states by fitting experimental photoluminescence data. We will concentrate on the specific example of an $A l_{0.3} G a_{0.7} A s$ surface with a nearby $G a A s$ quantum well [1],8]. At energy close to the bottom of the $e 1$ band of well 2 the $A l_{0.3} G a_{0.7} A s$ surface has only donor-like states belonging to the exponentially vanishing Urbach tail

$$
\nu_{e}^{(1)}(\epsilon)=\frac{m_{e}^{(1)}}{\pi \hbar^{2}} \exp \left(-\frac{\Delta E_{c}+e F b-E_{e 1}^{(2)}-\epsilon}{\epsilon_{e}}\right) .
$$

Such states are assumed to be nodal hydrogenic wavefunctions [19] with radius $r_{e}$ fixed by the depth into the gap. Their explicit expression is given in Appendix B. We assume that at the top of the gap the state density is the two dimensional density of free $A l_{0.3} G a_{0.7} A s$ electrons with effective mass $m_{e}^{(1)}$. The parameter $\epsilon_{e}$ will be considered as a fitting parameter. According to Eq. (17) and Eq. (12) where we can assume $E_{e 1}^{(1)}=-\infty$, the Fermi energy for the donor-like surface band is

$$
\epsilon_{F}^{(1)}=\Delta E_{c}+e F b-E_{e 1}^{(2)}+\epsilon_{e} \ln \left(\frac{\pi \hbar^{2} n_{1}}{m_{e}^{(1)} \epsilon_{e}}\right) .
$$

On the other hand, at energy close to the bottom of the $h h 1$ of well 2 the $A l_{0.3} G a_{0.7} A s$ surface has a very high concentration of acceptor-like defect states [18]. We schematize them again by nodal hydrogenic wavefunctions [19] but with radius $r_{h}$ to be considered as a second fitting parameter. These states are assumed to be distributed in energy with constant density $\nu_{h}^{(1)}$. The Fermi energy for the acceptor-like surface band is then

$$
\epsilon_{F}^{(1)}=p_{1} / \nu_{h}^{(1)}+E_{h h 1}^{(1)}+e F b-E_{h h 1}^{(2)} .
$$

The tunneling matrix elements for the above surface states and surface densities are evaluated explicitly in Appendix B.

According to the experiment reported in [1] we choose the well width $a_{2}=60 \AA$ the temperature $T=4.2 \mathrm{~K}$, the photon energy $h \nu=1.608 \mathrm{eV}$ and the incident power density $P_{i}=0.5 \mathrm{Wcm}^{-2}$. The incident efficiency is estimated to be $1 \%$ and we take $G_{2}=0.01 P_{i} / h \nu$. The relevant material parameters are [20]: $\Delta E_{c}=0.3 \mathrm{eV}, \Delta E_{v}=0.128 \mathrm{eV}$, $m_{e}^{(1)}=0.091 m, m_{e}^{(2)}=0.067 m, m_{h}^{(2)}=0.34 m, m$ being the free electron mass, and $\varepsilon_{r}=12$. Moreover we put $\lambda_{2}=6 \mathrm{~cm}^{2} \mathrm{~s}^{-1}[14]$. We assume an acceptor-like surface state density $\nu_{h}^{(1)}=10^{14} \mathrm{~cm}^{-2} \mathrm{eV}^{-1}$ [21] with $E_{h h 1}^{(1)} \simeq 1 \mathrm{eV}$ (the results we found do not depend crucially on this particular value). The free parameters, $\epsilon_{e}$ and $r_{h}$, are fixed by fitting the normalized photoluminescence intensity $I_{2} / I_{2 \infty}$ to the experimental data 11] obtained for different values of the barrier width $b$. A least-square-error procedure gives the unique solution $\epsilon_{e}=12 \mathrm{meV}$ and $r_{h}=11 \AA$. In Fig. 5 , comparison is made between the ratio $I_{2} / I_{2 \infty}$, calculated with these values, and the experimental data.

Due to presence of two parameters the agreement between theoretical and experimental data in Fig. 5 should be considered as a source of information for these two parameters in the case the model is valid more than a proof of validity of the model itself. A reliable check of validity of our model is obtained by comparing the theoretical values of the self-consistently estimated electric field $F$ with the values deduced from measured Stark shifts as a function of the QW excitation [8]. The calculated field values are shown in Fig. 6 as a function of the barrier thickness $b$, for different values of the incident power. It is seen that, for high levels of excitation, the field approaches values of order $10^{5} \mathrm{~V} \mathrm{~cm}^{-1}$ and keeps increasing when the barrier becomes thinner. Direct comparison with experiment is made for a 80 - $\AA$-thick barrier, a choice dictated by the wide laser power range where data were available for this moderately tunneling sample. To obtain the value of the electric field from the measured Stark shift $\Delta E_{p}$ we use the relationship $\Delta E_{p}=K F^{3 / 2}$, empirically deduced from known results in the literature [22,23. The constant $K$ is fixed by imposing that the electric field obtained from the measured Stark shift $\Delta E_{p}=0.33 \mathrm{meV}$, corresponding to an incident power 
density $P_{i}=0.68 \mathrm{~W} \mathrm{~cm}^{-2}$, coincides with the calculated value. We obtain $K=1.25 \cdot 10^{-7} \mathrm{meV}\left(\mathrm{V} \mathrm{cm}^{-1}\right)^{-3 / 2}$, which compares well with the values extracted from the data of Ref. [22], $\left.K \simeq 1 \cdot 10^{-7} \mathrm{meV}(\mathrm{V} \mathrm{cm})^{-1}\right)^{-3 / 2}$, and Ref. [23], $K \simeq 0.6 \cdot 10^{-7} \mathrm{meV}\left(\mathrm{V} \mathrm{cm}^{-1}\right)^{-3 / 2}$.

The comparison between the calculated and experimentally deduced electric field is shown in Fig. 7 as a function of the incident power density. The agreement is very good for a power range extending over 3 orders of magnitude. The near-coincidence of the scale factor $K$ with other independently obtained values should not be attributed much importance, on one side because of the approximations used in the model, e.g. density and distribution of surface states, and, on the other side, because of the experimental Stark shift is observed in the luminescence from the quantum well, where the field is non-uniform and possibly different from its value in the barrier [8]. Instead, the correct functional dependence of the field on the excitation level is a clear confirmation that the self-consistent approach provides a reasonably accurate description of the whole process.

\section{CONCLUSIONS}

The photoluminescence efficiency in asymmetric double quantum wells and in near-surface quantum wells is strongly influenced by the tunneling of both electrons and holes between the two wells, or the well and the surface states. The theoretical model presented here takes into account this effect and allows a quantitative prediction for the photoluminescence rates and the value of the electric field which needs to be established across the tunneling barrier in a steady-state situation. Experimental results regarding both ADQW and near-surface QWs, namely changes in photoluminescence efficiency and peak shifts due to the self-induced electric field, are very well accounted for.

Our model can be readily generalized to include the case of an externally applied electric field as well as the case of not constant excitation.

\section{ACKNOWLEDGMENTS}

We thank M. Capizzi, B. Bonanni and M. Colocci for very profitable discussions.

\section{APPENDIX A: ADQW MATRIX ELEMENTS}

We call $z$ the coordinate orthogonal to the interfaces and suppose that the quantum well 1 is in $-a_{1} \leq z \leq 0$ and the quantum well 2 in $b \leq z \leq b+a_{2}$. Firstly let us consider the case of electrons. As schematized in Fig. 8 we assume a rectangular potential profile with left and right discontinuities $V_{l}^{(1)}=\Delta E_{c}$ and $V_{r}^{(1)}=\Delta E_{c}-e F b / 2$ for

the well 1 and $V_{l}^{(2)}=\Delta E_{c}+e F b / 2$ and $V_{r}^{(2)}=\Delta E_{c}$ for the well 2 where $F$ is the electric field in the barrier region $0 \leq z \leq b$. The electron wavefunctions at energy $\epsilon$, measured from the bottom of the $e 1$ band of well 2 , are

$$
\Phi_{\epsilon}^{(1)}(x, y, z)=\frac{e^{i k_{x}^{(1)} x+i k_{y}^{(1)} y}}{\sqrt{A}} C^{(1)} \begin{cases}\sin \delta^{(1)} e^{k_{l}^{(1)}(z+a 1)} & z<-a_{1} \\ \sin \left[k^{(1)}\left(z+a_{1}\right)+\delta^{(1)}\right] & -a_{1}<z<0 \\ \sin \left[k^{(1)} a_{1}+\delta^{(1)}\right] e^{-k_{r}^{(1)} z} & 0<z\end{cases}
$$

where

$$
\begin{gathered}
\frac{\hbar^{2}}{2 m_{e}^{(1)}}\left[\left(k_{x}^{(1)}\right)^{2}+\left(k_{y}^{(1)}\right)^{2}\right]=\epsilon+E_{e 1}^{(2)}-E_{e 1}^{(1)}-e F b \\
k_{l}^{(1)}=\sqrt{\frac{2 m_{e}^{(1)}}{\hbar^{2}}\left(\Delta E_{c}-E_{e 1}^{(1)}\right)} \\
k^{(1)}=\sqrt{\frac{2 m_{e}^{(1)}}{\hbar^{2}} E_{e 1}^{(1)}}
\end{gathered}
$$




$$
k_{r}^{(1)}=\sqrt{\frac{2 m_{e}^{(1)}}{\hbar^{2}}\left(\Delta E_{c}-e F b / 2-E_{e 1}^{(1)}\right)}
$$

for the well 1 and

$$
\Phi_{\epsilon}^{(2)}(x, y, z)=\frac{e^{i k_{x}^{(2)} x+i k_{y}^{(2)} y}}{\sqrt{A}} C^{(2)} \begin{cases}\sin \delta^{(2)} e^{k_{l}^{(2)}(z-b)} & z<b \\ \sin \left[k^{(2)}(z-b)+\delta^{(2)}\right] & b<z<b+a_{2} \\ \sin \left[k^{(2)} a_{2}+\delta^{(2)}\right] e^{-k_{r}^{(2)}\left(z-b-a_{2}\right)} & b+a_{2}<z\end{cases}
$$

where

$$
\begin{gathered}
\frac{\hbar^{2}}{2 m_{e}^{(2)}}\left[\left(k_{x}^{(2)}\right)^{2}+\left(k_{y}^{(2)}\right)^{2}\right]=\epsilon \\
k_{l}^{(2)}=\sqrt{\frac{2 m_{e}^{(2)}}{\hbar^{2}}\left(\Delta E_{c}+e F b / 2-E_{e 1}^{(2)}\right)} \\
k^{(2)}=\sqrt{\frac{2 m_{e}^{(2)}}{\hbar^{2}} E_{e 1}^{(2)}} \\
k_{r}^{(2)}=\sqrt{\frac{2 m_{e}^{(2)}}{\hbar^{2}}\left(\Delta E_{c}-E_{e 1}^{(2)}\right)}
\end{gathered}
$$

for the well 2. Energy $E_{e 1}^{(i)}$ measures the bottom of the $e 1$ band in the well $i=1,2$ from the bottom of the same well and is determined by solving

$$
k^{(i)} a_{i}=\pi-\sin ^{-1}\left(\frac{\hbar k^{(i)}}{\sqrt{2 m_{e}^{(i)} V_{l}^{(i)}}}\right)-\sin ^{-1}\left(\frac{\hbar k^{(i)}}{\sqrt{2 m_{e}^{(i)} V_{r}^{(i)}}}\right) .
$$

The phase shifts are $\delta^{(i)}=\tan ^{-1}\left(k^{(i)} / k_{l}^{(i)}\right), i=1,2$. The constants $C^{(i)}, i=1,2$, are fixed by normalizing the wavefunctions $\Phi_{\epsilon}^{(i)}$ :

$$
C^{(i)}=\left[\frac{\sin ^{2} \delta^{(i)}}{2 k_{l}^{(i)}}+\frac{\sin ^{2}\left(k^{(i)} a_{i}+\delta^{(i)}\right)}{2 k_{r}^{(i)}}+\frac{a_{i}}{2}-\frac{\sin \left[2\left(k^{(i)} a_{i}+\delta^{(i)}\right)\right]-\sin \left(2 \delta^{(i)}\right)}{4 k^{(i)}}\right]^{-1 / 2} .
$$

For $\epsilon=0$ and with the assumption that $V_{e}$ in Eq. (6) vanishes everywhere but in the barrier region where $V_{e}=\Delta E_{c}$, the tunneling matrix element is

$$
\begin{aligned}
& \left\langle\Phi_{0}^{(1)}\left|V_{e}\right| \Phi_{0}^{(2)}\right\rangle= \\
& \int_{0}^{\sqrt{A}} d x \int_{0}^{\sqrt{A}} d y \int_{0}^{b} d z \frac{e^{-i k_{x}^{(1)} x-i k_{y}^{(1)} y}}{\sqrt{A}} C^{(1)} \sin \left[k^{(1)} a_{1}+\delta^{(1)}\right] e^{-k_{r}^{(1)} z} \Delta E_{c} \frac{1}{\sqrt{A}} C^{(2)} \sin \delta^{(2)} e^{k_{l}^{(2)}(z-b)} \\
& =\frac{C^{(1)} C^{(2)}}{A} \sin \left[k^{(1)} a_{1}+\delta^{(1)}\right] \sin \delta^{(2)} \frac{e^{-k_{r}^{(1)} b}-e^{-k_{l}^{(2)} b}}{k_{l}^{(2)}-k_{r}^{(1)}} \frac{1-e^{-i k_{x}^{(1)} \sqrt{A}}}{i k_{x}^{(1)}} \frac{1-e^{-i k_{y}^{(1)} \sqrt{A}}}{i k_{y}^{(1)}} .
\end{aligned}
$$

The last two factors in this expression quickly oscillates with $k_{x}^{(1)}$ and $k_{y}^{(1)}\left(k_{x}^{(1)}\right)=\sqrt{2 m_{e}^{(1)}\left(E_{e 1}^{(2)}-E_{e 1}^{(1)}-e F b\right) / \hbar^{2}-\left(k_{x}^{(1)}\right)^{2}}$. By taking the value for $k_{x}^{(1)}=0$ (maximum value) the electron tunneling matrix element is estimated as

$$
\left|\left\langle\Phi_{0}^{(1)}\left|V_{e}\right| \Phi_{0}^{(2)}\right\rangle\right| \simeq\left|\frac{2 \hbar \Delta E_{c} C^{(1)} C^{(2)} \sin \left[k^{(1)} a_{1}+\delta^{(1)}\right] \sin \delta^{(2)}}{\sqrt{2 m_{e}^{(1)} A\left(E_{e 1}^{(2)}-E_{e 1}^{(1)}-e F b\right)}} \frac{e^{-k_{r}^{(1)} b}-e^{-k_{l}^{(2)} b}}{k_{l}^{(2)}-k_{r}^{(1)}}\right| .
$$

In the case of holes we have a completely analogous situation where the relevant band is $h h 1$ instead of $e 1$. The expressions derived for electrons still hold for holes with the substitutions $m_{e}^{(i)} \rightarrow m_{h}^{(1)}, i=1,2, \Delta E_{c} \rightarrow \Delta E_{v}$, $e F b \rightarrow-e F b$. 


\section{APPENDIX B: NEAR-SURFACE QW MATRIX ELEMENTS}

Following the notations of Appendix A, the surface is defined by the plane $z=0$ and the well 2 is in $b<z<b+a_{2}$. Firstly, we consider the case of electrons and we measure the energy from the bottom of the $e 1$ band of well 2 . The state $\Phi_{\epsilon}^{(2)}(x, y, z)$ is given by Eq. (A6). The donor-like surface state $\Phi_{\epsilon}^{(1)}(x, y, z)$ is approximated by a truncated $2 p$ hydrogenic wavefunction [19]

$$
\Phi_{\epsilon}^{(1)}(x, y, z)=\frac{z}{4 \sqrt{\pi} r_{e}^{5 / 2}} e^{r / 2 r_{e}} \begin{cases}0 & z<0 \\ 1 & 0<z\end{cases}
$$

where $r=\sqrt{x^{2}+y^{2}+z^{2}}$. The state is at energy $\hbar^{2} /\left(8 m_{e}^{(1)} r_{e}^{2}\right)$ below the bottom of the conduction band for the barrier material where the electron effective mass is $m_{e}^{(1)}$. By imposing this energy to correspond to $\epsilon$ we determine the radius $r_{e}$

$$
r_{e}=\frac{\hbar}{\sqrt{8 m_{e}^{(1)}\left(\Delta E_{c}-E_{e 1}^{(2)}+e F b-\epsilon\right)}}
$$

Using parabolic coordinates $\eta=(r-z) / r_{e}, \xi=(r+z) / r_{e}, \varphi=\tan ^{-1}(y / x)$, the tunneling matrix element between the well and surface states at $\epsilon=0$ is:

$$
\begin{aligned}
& \left\langle\Phi_{0}^{(1)}\left|V_{e}\right| \Phi_{0}^{(2)}\right\rangle= \\
& \int_{0}^{\infty} d \eta \int_{\eta}^{\eta+2 b / r_{e}} d \xi \int_{0}^{2 \pi} d \varphi \frac{r_{e}^{3}(\xi+\eta)}{4} \frac{(\xi-\eta) e^{-(\xi+\eta) / 4}}{8 \sqrt{\pi} r_{e}^{3 / 2}} \Delta E_{c} \frac{C^{(2)} \sin \delta^{(2)} e^{k_{l}^{(2)}\left[(\xi-\eta) r_{e} / 2-b\right]}}{\sqrt{A}}= \\
& \frac{\sqrt{\pi} r_{e}^{3 / 2} \Delta E_{c} C^{(2)} \sin \delta^{(2)}}{8 \sqrt{A}}\left\{e^{-k_{l}^{(2)} b\left[\frac{1}{4\left(2 k_{l}^{(2)} r_{e}-1\right)^{2}}-\frac{1}{32\left(2 k_{l}^{(2)} r_{e}-1\right)^{3}}\right]+}\right. \\
& \left.e^{-b / 2 r_{e}}\left[\frac{\left(b / r_{e}\right)^{2}+2 b / r_{e}}{2 k_{l}^{(2)} r_{e}-1}-\frac{1+b / r_{e}}{4\left(2 k_{l}^{(2)} r_{e}-1\right)^{2}}+\frac{1}{32\left(2 k_{l}^{(2)} r_{e}-1\right)^{3}}\right]\right\}
\end{aligned}
$$

In the case of holes we have a completely analogous situation where the relevant band of well 2 is $h h 1$ instead of $e 1$ and the acceptor-like surface state is given by Eq. (B1) with $r_{e} \rightarrow r_{h}$. Equation (B3) gives the tunneling matrix element for holes with the substitutions $r_{e} \rightarrow r_{h}, \Delta E_{c} \rightarrow \Delta E_{v}, e F b \rightarrow-e F b$.

[1] Y. L. Chang, I. H. Tan, Y. H. Zhang, J. Merz, E. Hu, A. Frova, and V. Emiliani, Appl. Phys. Lett. 62, 2697 (1993).

[2] J. M. Moison, K. Elcess, F. Houzay, J. Y. Marzin, J. M. Gerard, F. Barthe, and M. Bensoussan, Phys. Rev. B 41, 12945 (1990).

[3] Z. Sobiesierski, D. I. Westwood, H. Fujikura, T. Fukui, and H. Hasegawa, J. Vac. Sci. Technol. B 11, 7010 (1993).

[4] V. N. Astratov, and Yu. A. Vlasov, in Proceeding of ICDS-17 Conference, 18-23 July,1993, Gmunden (Austria).

[5] T. B. Norris, N. Vodjdani, B. Vinter, E. Costard, and E. Böckenhoff, Phys. Rev. B 43, 1867 (1991).

[6] F. Capasso, K. Mohammed, A. Y. Cho, R. Hull, and A. L. Hutchinson, Appl. Phys. Lett. 47, 420 (1985).

[7] M. Nido, M. G. Alexander, W. W. Rühle, T. Schweizer, and K. Köhler, Appl. Phys. Lett. 56, 355 (1990).

[8] V. Emiliani, B. Bonanni, C. Presilla, M. Capizzi, A. Frova, Y. L. Chang, I. H. Tan, J. L. Merz, M. Colocci, and M. Gurioli, J. Appl. Phys. 75, 5114 (1994).

[9] R. Sauer, K. Thonke, and W. T. Tsang, Phys. Rev. Lett. 61, 609 (1988).

[10] Ph. Roussignol, A. Vinattieri, L. Carraresi, M. Colocci, and A. Fasolino, Phys. Rev.B 44, 8873 (1991).

[11] M. Colocci, M. Gurioli, A. Vinattieri, F. Fermi, C. Deporis, J. Massaies, and G. Neu, Europhys. Lett. 12, 417 (1991).

[12] D. A. B. Miller, D. S. Chemla, T. C. Damen, A. C. Gossard, W. Wiegmann, T. H. Wood, and C. A. Burrus, Phys. Rev. Lett. 53, 2173 (1984).

[13] R. Ferreira, P. Rolland, Ph. Roussignol, C. Delande, A. Vinattieri, L. Carraresi, M. Coloccci, N. Roy, B. Sermage, J. F. Palmier, and B. Etienne, Phys. Rev. B 45, 11782 (1992).

[14] R. Strobel, R. Eccleston, J. Kuhl, and K. Köhler, Phys. Rev. B 43, 12564 (1991). 
[15] R. Nagarajan, M. Ishikawa, T. Fukushima, R. S. Geels, and J. E. Bowers, IEEE Journal of Quantum Electronics, 28, 1990 (1992).

[16] L. D. Landau and E. M. Lifšits, Quantum Mechanics, Nonrelativistic Theory (Pergamon Press, Oxford, 1958).

[17] Landolt-Börnstein Numerical Data and Functional Relationships in Science and Technology, Volume 17 Semiconductors, O. Madelung, M. Schults, and H. Weiss Editors (Springer-Verlag, Berlin 1982).

[18] W. E. Spicer, Z. Liliental-Weber, E. Weber, N. Newman, T. Kendelewicz, R. Cao, C. McCants, P. Mahowald, K. Miyano, and I. Lindau, J. Vac. Sci. Tech. B 6, 1245 (1988).

[19] J. D. Levine, Phys. Rev. 140, 586 (1965).

[20] R. Atanasov and F. Bassani, Solid State Commun. 84, 71 (1992).

[21] W. E. Spicer, P. W. Chye, P. R. Skeath, C. Y. Su, and I. Lindau, J. Vac. Sci. Tech. 16, 1422 (1979).

[22] E. O. Goebel, in Springer Proc. Phys., Vol. 25 (Springer, Berlin, 1988), pp. 204-217.

[23] L. Viña, R. T. Collins, E. E. Mendez, L. L. Chang, and L. Esaki, in Springer Proc. Phys., Vol. 25 (Springer, Berlin, 1988), pp. 230-243.

FIG. 1. Calculated emission intensity ratio $I_{1} / I_{2}$ from two coupled asymmetric wells $v s$ absorbed power density $P$ for various values of the barrier width $b$.

FIG. 2. Calculated electric field $F$ which establishes between the two wells, in the same cases of Fig. 1.

FIG. 3. Calculated normalized photoluminescence intensities $I_{1} / I_{1 \infty}$ and $I_{2} / I_{2 \infty} v s$ barrier thickness $b$ for different absorbed power densities $P$. $I_{i \infty}$ is the photoluminescence intensity from well $i$ in the limit $b \rightarrow \infty$.

FIG. 4. Calculated electric field $F$ which establishes between the two wells in the same cases of Fig. 3.

FIG. 5. Normalized photoluminescence ratio $I_{2} / I_{2 \infty}$ of a near-surface well $v s$ the surface-barrier thickness $b$. Dots: experimental data from Ref. [1]; solid line: best fitting in terms of the self-consistent model. Incident power density is $P_{i}=0.5 \mathrm{~W}$ $\mathrm{cm}^{-2}$.

FIG. 6. Calculated electric field $F$ across the surface barrier $v s$ the surface barrier thickness $b$ for different incident power densities $P_{i}$.

FIG. 7. Comparison between the electric field $F$ calculated from the model (solid line) and deduced from the measured Stark shifts [8] (dots) vs the incident power density $P_{i}$. The surface-barrier thickness is $\mathrm{b}=80 \AA$.

FIG. 8. Energy-space diagram for electrons in the asymmetric double quantum well. 